\title{
Operação de Mustard no tratamento cirúrgico da transposição simples das grandes artérias
}

\author{
Cláudio A. GOMES * , Jorge Vieira RODRIGUES * Fernando MORAES NETO *, Cleuza Lapa \\ SANTOS *, Sandra MATTOS *, Carlos R. MORAES *
}

RBCCV $44205-270$

GOMES, C. A.; RODRIGUES, J. V.; MORAES NETO, F.; SANTOS, C. L.; MATTOS, S.; MORAES, C. R. Operaçâo de Mustard no tratamento cirúrgico da transposiçăo simples das grandes artérias. Rev. Bras. Cir. Cardiovasc., 10 (3): 139-143, 1995.

RESUMO: Entre abril de 1975 e janeiro de 1994, 28 crianças foram submetidas à operaçảo de Mustard para tratamento da transposição simples das grandes artérias através de modificaçāo técnica destinada a evitar arritmias e minimizar o problema da obstruçăo venosa sistémica ou pulmonar. A idade variou de 21 dias a 7 anos (média, 18 meses). A maioria dos pacientes (22) estava no primeiro ano de vida. Ocorreram seis óbitos no período de internaçâo hospitalar, quatro dos quais em crianças com menos de quatro meses de idade. Entre os 22 sobreviventes, năo foram detectadas arritmias graves ou disfunçăo direita. Trës pacientes foram reoperados com sucesso, para tratamento de obstrução venosa de veia cava superior (2 casos) e veias pulmonares ( 1 caso). Os resultados atuais da operaçāo de Mustard modificada são bons, e este procedimento continua como alternativa válida em pacientes com tran sposiçáo simples das grandes artérias náo candidatos à correçāo anatômica.

DESCRITORES: Transposiçăo das grandes artérias, cirurgia. Mustard, operaçăo de.

\section{INTRODUÇÃO}

O tratamento cirúrgico da transposição das grandes artérias (TGA) teve início em 1950, quando BLALOCK \& HANLON ${ }^{4}$ descreveram a técnica de septectomia atrial techada. A criação de uma ampla comunicação interatrial, permitindo a mistura das duas circulaçōes, possibilitou a sobrevida até idade mais avançada de muitas crianças portadoras dessa malformaçāo. Em 1953, LILLEHEI \& VARCO 10 descreveram uma técnica de correçăo fisiológica parcial, que consistia na anastomose das veias pulmonares direitas com o átrio direito e da veia cava inferior com o átrio esquerdo. BAFFES ${ }^{3}$, em 1956, incorporou a utilização de um homoenxerto aórtico para conectar a veia cava inferior ao átrio esquerdo.
O conceito de correçăo fisiológica total a nível atrial, por remodelamento da parede do átrio direito e do septo interatrial, foi inicialmente proposto por ALBERT 1 , em 1954. A primeira operaçāo desse tipo foi realizada por SENNING ${ }^{18}$, em 1959. A operaçāo de Senning mostrou, de inicio, alta mortalidade e somente foi revivida e utilizada em larga escala após os trabalhos de QUAEJEBEUR et alii ${ }^{15}$, em 1977. Em 1964, MUSTARD ${ }^{13}$ descreveu uma nova técnica de correçăo atrial da TGA, que consiste na excisão completa do septo interatrial e na utilização de um enxerto de pericárdio, a fim de redirecionar o sangue das veias cavas para a valva mitral e ventrículo esquerdo e o sangue das veias pulmonares para a valva tricúspide e o ventrículo direito. Esse tipo de técnica foi, por muitos anos, a operação mais utilizada na correção da TGA.

\footnotetext{
Trabalho realizado no Hospital das Clínicas da Universidade Federal de Pernambuco e no Instituto do Coraçăo de Pernambuco. Recite, PE, Brasil. Recebido para publicação em setembro de 1995.

* Do Hospital das Clinicas da Universidade Federal de Pernambuco e/ou Instituto do Coraçăo de Pernambuco.

Endereço para correspondência: Cláudio A. Gomes - Av, Portugal, 163. CEP 52.010-010 Recile, PE, Brasil
} 
Um grande progresso no tratamento da TGA ocorreu a partir de 1966, quando RASHKIND \& MILLER 17 descreveram a septostomia com balāo. Essa técnica permitiu a paliação em neonatos, postergando-se a correção atrial para depois do $4^{\circ}$ mês de vida.

Em 1975, a contribuiçăo mais importante na história da cirurgia da TGA foi dada por JATENE et alii ${ }^{8}$, ao conseguirem a primeira sobrevida com a correção anatômica a nivel arterial. De início, a operação de Jatene foi reservada a casos de TGA com comunicação interventricular (CIV), mas YACOUB et alii ${ }^{22}$, em 1977, passaram a realizá-la em casos de TGA simples, após preparação do ventrículo esquerdo através de cerclagem do tronco pulmonar e shunt sistêmico-pulmonar. Outro grande avanço foi dado por CASTANEDA et alii ${ }^{5}$, em 1984, e por QUAEJEBEUR et alii ${ }^{16}$, em 1986, ao demonstrarem que a operação de Jatene poderia ser aplicada em casos de TGA simples, desde que fosse feita nas primeiras semanas de vida, ou seja, antes da queda da resistência arterial pulmonar e da diminuição da massa do ventrículo esquerdo.

No presente trabalho revemos nossa experiência com 28 crianças submetidas à operaçăo de Mustard.

\section{CASUISTICA E MÉTODOS}

No período entre abril de 1975 e janeiro de 1994, 28 crianças portadoras de TGA simples foram operadas pela técnica de Mustard, em nosso Serviço. Dezessete $(60,7 \%)$ eram do sexo masculino e $11(39,3 \%)$, do feminino, variando a idade de 21 dias a 7 anos (média de 18 meses). $O$ peso variou de 3,4 a $23 \mathrm{~kg}$ (média de $7,8 \mathrm{~kg}$ ).

Todos os pacientes foram submetidos a completo estudo clínico, radiológico e eletrocardiográfico, sendo o diagnóstico definitivo obtido através de estudo hemodinâmico em 22 pacientes. Nas outras 6 crianças, a cirurgia foi indicada apenas com diagnóstico ecocardiográfico e em 12 realizou-se septostomia com balão.

\section{TÉCNICA CIRÚRGICA}

Utilizou-se perfusāo com auxilio de hipotermia profunda $\left(18^{\circ} \mathrm{C}\right)$ em 19 casos, realizando-se parada circulatória total em 13 e hipofluxo em 6 . Os outros 9 foram operados com circulaçāo extracorpórea convencional a $30^{\circ} \mathrm{C}$. Obteve-se proteçăo miocárdica por infusão de soluçăo cardioplégica gelada na aorta ascendente e hipotermia tópica do coraçāo.
A circulação extracorpórea foi estabelecida através de cânula na aorta ascendente. Nos casos em que se realizou parada circulatória, colocou-se apenas uma cânula venosa através da aurícula direita. Nos demais, a introdução da cânula na veia cava superior foi através da auricula direita e a veia cava inferior, bem próximo ao diafragma. Durante as manobras das cavas com cadarços, evitou-se traumatizar a junçăo da veia cava superior com o átrio direito.

A operação de Mustard foi sempre realizada seguindo-se as modificaçōes levadas a efeito no Brompton Hospital 20. A atriotomia direita é curva, estendendo-se da veia cava inferior ao topo da aurícula direita. O septo interatrial é amplamente ressecado e suas bordas, reendotelizadas. As medidas para confecçāo do enxerto sāo, entāo, tomadas: primeiro, a distância entre as veias pulmonares esquerdas e o remanescente do septo interatrial e, depois, os diâmetros dos orifícios das veias cavas. $O$ enxerto é cortado em forma de "calça", tal como descrito por QUAEJEBEUR \& BROM ${ }^{14}$. Utilizamos sempre dura-máter homóloga ou pericárdio bovino.

$O$ enxerto é suturado seguindo os princípios básicos de MUSTARD ${ }^{13}$, mas com a parte periférica (borda externa da "calça") colocada bem longe dos orificios das veias cavas.

Nas áreas mais próximas do orifício das cavas, o enxerto é incorporado às bordas da atriotomia. Procura-se evitar, dessa maneira, lesão do nó sinoatrial e sua artéria, seja por hematoma ou fibrose tardia. A parte inferior da linha de sutura passa posterior ao seio coronário, drenado para o átrio sistêmico (Figura 1).

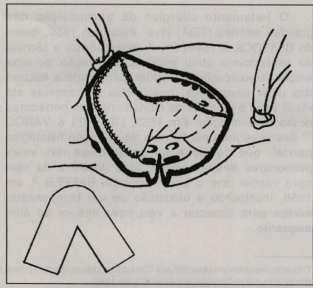

Fig. 1 - Esquema da técnica de atrioseptoplastia com o enxerto em forma de "calça". 


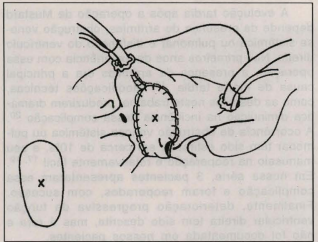

Fig. 2 - Técnica de ampliação do novo átrio esquerdo.

Na maioria dos pacientes, procedeu-se ao alargamento do átrio sistèmico (Figura 2). Para isso, a atriotomia é estendida em direçāo à parede posterior do átrio esquerdo, separando-se as veias pulmonares direitas. Utiliza-se um enxerto para a ampliaçăo dessa região.

\section{RESULTADOS}

\section{Mortalidade Imediata}

Seis $(21,4 \%)$ pacientes faleceram no período de internação hospitalar, em decorrência de síndrome de baixo débito ( 3 casos), sepsis ( 1 caso), pulmão de choque ( 1 caso) e falência aguda do ventriculo direito ( 1 caso). Observou-se nitida relaçāo entre a mortalidade e a idade na época da cirurgia (Tabela 1). Das 4 crianças operadas no $1^{\circ}$ mês de vida, $2(50 \%)$ faleceram. De 7 operadas entre o $1^{\circ}$ e o $4^{\circ}$ mès, $2(28,5 \%)$ morreram. $E$, finalmente, de 17 operadas com mais de quatro meses, apenas $2(11,7 \%)$ vieram a falecer.

\section{Complicações Pós-Operatórias}

Além dos pacientes que faleceram, 7 outros exibiram complicaçōes pós-operatórias significativas, a saber: tamponamento cardiaco (2 casos), lesāo neurológica discreta ( 2 casos), osteomielite do esterno ( 1 caso), infecçăo pulmonar ( 1 caso) e insuficiência renal aguda ( 1 caso).

\section{Evolução Tardia}

Nenhum dos sobreviventes exibiu arritmias sig- nificativas na evolução tardia. Dois apresentaram obstrução de veia cava superior e 1 , de veias pulmonares. Os 2 primeiros pacientes foram operados com dois e quatro meses de vida e o terceiro, com onze meses. Todos os 3 foram reoperados com sucesso, porém 1 faleceu no $3^{\circ}$ ano de pós-operatório, devido a cirrose hepática.

\section{COMENTÁRIOS}

Atualmente, parece evidente que a forma ideal de tratamento de crianças portadoras de TGA simples é a correçäo anatômica a nível arterial (operação de Jatene), no período neonatal, antes da reduçăo da massa do ventrículo esquerdo em decorrência da queda da resistència arterial pulmonar $5,16$. BACKER et alii ${ }^{2}$, entre outros, demonstraram que, embora a mortalidade operatória e tardia das operaçōes de Jatene e de Mustard seja comparável, o primeiro procedimento, a longo prazo, mostra resultados superiores em termos de arritmias e melhor preservação da função ventricular.

Também parece evidente que a correçăo a nivel arterial é procedimento mais complexo do que as operaçōes de correção a nivel atrial. Disso resulta que nem sempre é possivel realizar a forma de tratamento ideal da TGA simples por duas razōes principais: primeiro, existem dificuldades institucionais no manuseio de neonatos com cardiopatias graves; segundo, mesmo instituiçőes capacitadas recebem, com frequeência, crianças que já perderam a oportunidade da correçăo anatômica, ou por terem sido submetidas a septostomia com balăo, ou por já terem nascido com comunicação interatrial que lhes permitiu sobreviver, sendo o diagnóstico feito mais tardiamente. Para esse grupo de doentes, resta a correção a nivel atrial, visto que a técnica de preparação do ventrículo esquerdo, proposta por YACOUB et alii ${ }^{22}$, não demonstrou, até o momento, vantagens $\Theta$ aplicabilidade prática em larga escala. E, portanto, válida a análise dos resultados das técnicas de correção atrial da TGA simples.

Até 1977, a operação de Mustard era usada, na maioria das instituições, para correção da TGA simples $19,20,21$. A evolução, a longo prazo, dos sobre-

TABELA 1

RELAÇAOO IDADE /MORTALIDADE

\begin{tabular}{lccr}
\hline Idade & NoCasos & Óbitos & $\%$ \\
\hline < 30 dias & 4 & 2 & 50,0 \\
1 a 4 meses & 7 & 2 & 28,5 \\
4 a 12 meses & 11 & 1 & 9,0 \\
> 12 meses & 6 & 1 & 16,6 \\
\hline
\end{tabular}


GOMES, C. A.; RODRIGUES, J. V.; MORAES NETO, F.; SANTOS, C. L.; MATTOS, S.; MORAES, C. R. - Operaçāo de Mustard no tratamento cirúrgico da transposiçăo simples das grandes artérias. Rev. Bras. Cir. Cardiovasc., 10 (3): 139-143, 1995.

viventes mostrou uma inquietante incidência de morbidade e mortalidade relacionada a arritmias e obstrução venosa. Por conseguinte, a operaçăo de Senning foi revivida $11,15 \mathrm{e}$, sobretudo, importantes modificaçōes técnicas foram introduzidas na operação de Mustard 20 . As modificações propostas pelo grupo do Brompton Hospital, de Londres, e utilizadas neste trabalho, mudaram substancialmente os resultados.

Diversos trabalhos têm demonstrado que os fatores de risco determinantes da mortalidade hospitalar, na operaçāo de Mustard, sāo a baixa idade e o baixo peso? Isso ficou evidenciado em nossa experiência de 28 casos operados, em que ocorreram 4 das 6 mortes em crianças com menos de quatro meses de vida. Também verificamos, como tem sido descrito, que a causa mais freqüente de óbito é a síndrome de baixo débito, graças ao reduzido tamanho dos átrios, que passam a funcionar mais como um conduto do que como um reservatório, determinando baixa pressăo ventricular 9 .
A evolução tardia após a operação de Mustard depende da presença de arritmias, obstruçāo venosa sistêmica ou pulmonar e disfunçāo do ventrículo direito. Nos primeiros anos de experiência com essa operação, a presença de arritmias era a principal causa de morte tardia ${ }^{19}$. Modificaçöes técnicas, como as descritas neste trabalho, produziram dramática diminuiçăo na incidéncia dessa complicação 20 . A ocorrência de obstrução venosa sistêmica ou pulmonar tem sido estimada em cerca de $10 \%$, e seu manuseio na reoperação é relativamente fácil ${ }^{17}, 19$. Em nossa série, 3 pacientes apresentaram essa complicaçăo e foram reoperados, com sucesso. Finalmente, deterioração progressiva da função ventricular direita tem sido descrita, mas é rara e não foi documentada em nossos pacientes.

Os resultados da operação de Mustard são, portanto, bastante satisfatórios e esse procedimento continua alternativa válida no tratamento cirúrgico da TGA simples, especialmente em crianças com mais de 3 ou 4 meses de vida e para as quais a correçẫo anatômica nāo esteja indicada.

\section{RBCCV $44205-270$}

GOMES, C. A.; RODRIGUES, J. V.; MORAES NETO, F; SANTOS, C. L.; MATTOS, S.; MORAES, C. R. Mustard operation for surgical treatment of simple transposition of the great arteries. Rev. Bras. Cir. Cardiovasc., 10 (3): 139-143, 1995.

ABSTRACT: Between April of 1975 and January of 1994, 28 patients underwent correction of simple transposition of the great arteries by a modification of the Mustard operation, designed to avoid dysrhythmias and to minimize systemic and pulmonary venous obstruction. Age ranged from 21 days to 7 years (mean age 18 months). The majority (22 patients) were in the first year of life. Early mortality occurred in 6 patients. Four of the early death were in children younger than 4 months. Among the 22 survivors no serious dysthythmias or right ventricular dysfunction were detected. Three patients presented superior vena cava (2) and pulmonary (1) venous obstruction, and were easily managed at reoperation. Current results with the modified Mustard operation are good and this procedure is still an alternative in children with simple transposition who are not candidates for anatomical repair.

DESCRIPTORS: Transposition of the great arteries, surgery. Mustard operation.

\section{REFERÊNCIAS BIBLIOGRÁFICAS}

1 ALBERT, H. M. - Surgical correction of transposition of the great arteries. Surg. Forum, 5: 74-77, 1954.

2 BACKER C. L.; ILBAWI, M. N.; OHTAKE, S.; DELEON, S. Y.; MUSTER, A. J.; PAUL, M. H.; BENSON Jr., D. N.: IDRISS, F, S. - Transposition of the great arteries: a comparison of results of the Mustard procedure versus the arterial switch. Ann. Thorac. Surg., 48: 10-14, 1989.
3 BAFFES, T, G, - A new method for surgical correction of transposition of the aorta and pulmonary artery. Surg. Gynecol. Obstet, 102: 227-233, 1956.

4 BLALOCK, A. \& HANLON, C. R. - The surgical treatment of complete transposition of the aorta and pulmonary artery. Surg. Gynecol. Obstet., 90: 1-15, 1950.

5 CASTANEDA, A. R.; NORWOOD, W. I.; LANG, P.; SANDERS, S. P. - Transposition of great arteries and intact ventricular septum: anatomic correction in the neonate. Ann. Thorac. Surg., 38: 438-443, 1984. 
GOMES, C. A.; RODRIGUES, J. V.; MORAES NETO, F.; SANTOS, C. L.; MATTOS, S.; MORAES, C. R. - Operaçăo de Mustard no tratamento cirúrgico da transposiçăo simples das grandes artérias. Rov. Bras. Cir. Cardiovasc., 10 (3): 139-143, 1995.

6 CASTANEDA, A. R.; TRUSLER, G. A.; PAUL, M. H.; BLACKSTONE, E. H.; KIRKLIN, J. W. and the CONGENITAL HEART SURGEONS SOCIETY. The early results of treatment of simple transposition in the current era. J. Thorac. Cardiovasc. Surg., 95: $14-28,1988$.

7 COBANOGLU, A.; ABBRUZZESE, P. A.; FREIMANIS, I.; GARCIA, C. E.; GRUNKEMEIER, G.; STARR, A. - Pericardial baffle complications following the Mustard operation: age-related incidence and ease of management. J. Thorac. Cardiovasc. Surg., 87:371. 378,1984 .

8 JATENE, A. D.; FONTES, V. F; PAULISTA, P. P.; SOUZA, L. C. B.; NEGER, F.; GALANTIER, M.; SOUZA, J. E. M. R. - Successful anatomic correction of transposition of the great vessels: a preliminary report. Arq. Bras. Cardiol., 28: 461-464, 1975.

9 KIRKLIN, J. W. \& BARRATT-BOYES, B. G. - Cardlac surgery. 2. ed. New York, Churchil Livingstone, Inc., 1993. 1383 p. .

10 LILLEHEI, C. W. \& VARCO, R. L. - Certain physiologic, pathologic and surgical features of complete transposition of the great vessels. Surgery, 34:376$400,1953$.

11 MARX, G. R.; HOUGEN, J. J.; NORWOOD, W. I. FYLER, D. L.; CASTANEDA, A. R.; NADAS, A. S. Transposition of the great arteries with intact ventricular septum: results of Mustard and Senning operations in 123 consecutive patients. J. Am. Coll. Cardiol., 1: 476-483, 1983.

MORAES, C. R.; TOMPSON, G.; ARRUDA, M.; LAGRECA, J. R.; CAVALCANTI, I. L; VICTOR, E; ESCOBAR, M.; OLIVEIRA, M. D. - Correction of transposition of great arteries using homologous duramater: preliminary report. Vasc. Surg., 11: 19-25, 1977.

13 MUSTARD, W. T. - Successful two-stage correction of transposition of the great vessels. Surgery, 55:469$472,1964$.
14 QUAEgebeuf, J. M. \& BROM, A. G. - The trousershaped baffle for use in the Mustard operation. Ann. Thorac. Surg., 25: 240-242, 1978.

15 QUAEgebeur, J. M.; ROHMER, J.; BROM, A. G. Revival of the Senning operation in the treatment of transposition of the great arteries: preliminary report on recent experience. Thorax, 32: 517-524, 1977.

16 QUAEGEBEUR, J. M.; ROHMER, J.; OTTENKAMP, J.; TUIS, T.; KIRKLIN, J. W:; BLACKSTONE, E. H.; BROM, A. G. - The arterial switch operation: an eightyear experience. J. Thorac. Cardiovasc., Surg., 92: $361-384,1986$.

17 RASHKIND, W. J. \& MILLER, W. N. - Creation of an atrial septal defect without thoracotomy: a palliative approach to complete transposition of the great arteries. JAMA, 166: 991-992, 1966.

18 SENNING. A. - Surgical correction of transposition of the great vessels. Surgery, 45: 966-980, 1959

19 TRUSLER, G. A.; WILLIAMS, W. G.; IZUKAWA, T.; OLLEY, P. M. - Current results with the Mustard operation in isolated transposition of the great arteries. J. Thorac. Cardiovasc. Surg, 80: 381-389, 1980.

20 ULLAL, R. R.; ANDERSON, R. H.; LINCOLN, C. Mustard's operation moditied to avoid dysrhythmias and pulmonary and systemic venous obstruction. $J$. Thorac. Cardiovasc. Surg., 78: 431-439, 1979.

21 WILLIAMS, W. G.; TRUSLER, G. A.; KIRKLIN, J. W.; BLACKSTONE, E. H.; COLES, J. G.; IZUKAWA, T.; FREEDOM, R. M. - Early and late results of a protocol for simple transposition leading to an atrial (Mustard) repair. J. Thorac. Cardiovasc. Surg, 95: 717-726, 1988.

22 YACOUB, M. H.; RADLEY-SMITH, R.; MACLAURIN, R. - Two-stage operation for anatomical correction of transposition of the great arteries with intact ventricular septum. Lancet, 1: 1275-1278, 1977. 